\title{
Combining Execution Pipelines to Improve Parallel Implementation of HMMER on FPGA
}

\author{
Naeem Abbas ${ }^{\mathrm{a}, \mathrm{c}, \mathrm{e}}$, Steven Derrien ${ }^{\mathrm{a}, \mathrm{b}, \mathrm{e}}$, Sanjay Rajopadhye ${ }^{\mathrm{d}}$, Patrice Quinton ${ }^{\mathrm{c}}$, \\ Alexandre Cornu ${ }^{\mathrm{a}, \mathrm{e}}$, Dominique Lavenier ${ }^{\mathrm{a}, \mathrm{c}, \mathrm{e}}$ \\ ${ }^{a}$ IRISA-INRIA \\ ${ }^{b}$ Université de Rennes 1 \\ ${ }^{c}$ ENS Cachan Bretagne, Université Européenne de Bretagne, Campus de Ker Lann Avenue \\ Robert Schuman, 35170 Bruz, FRANCE. \\ ${ }^{d}$ Computer Science Department, Colorado State University, USA. \\ ${ }^{e}$ Campus de Beaulieu, 35000 Rennes, FRANCE.
}

\begin{abstract}
HMMER is a widely used tool in bioinformatic, based on the Profile Hidden Markov Models. The computation kernels of HMMER, namely MSV and P7Viterbi are very compute intensive, and their data dependencies if interpreted naively, lead to a purely sequential execution. In this paper, we propose an original parallelization scheme for HMMER by rewriting the mathematical formulation, to expose the hidden potential parallelization opportunities. Our parallelization scheme targets FPGA technology, and our architecture can achieve 10 times speedup compared with the latest HMMER3 SSE version, without compromising on the sensitivity of original algorithm.
\end{abstract}

Keywords:

\section{Introduction}

Sequence database homology searching is one of the most important application in computational molecular biology, where protein sequences of unknown characteristics are searched against database of known sequences in order to predict protein functions and to classify protein families. Profile Hidden Markov

\footnotetext{
* Corresponding author: currently at PNEC-NUST, Karachi, Pakistan.

Email address: abbas.naeem@pnec.nust.edu.pk (Naeem Abbas)
}

Preprint submitted to Journal of ${ }^{A} T_{E} X$ Templates

June 25, 2015

(C) 2015. This manuscript version is made available under the Elsevier user license http://www.elsevier.com/open-access/userlicense/1.0/ 
Models (HMMs), introduced to computational biology by Krogh et al. 1] for representing profiles of multiple sequence alignments, has been successfully applied in homology detection and protein classification $([2,[3,44)$. A profile HMM of the family is built from the multiple sequence alignment. For each column in the Profile HMM, a match state models the allowed residue, while an insert and delete state models the insertion of one or more residues, or the deletion of a residue. The multiple alignment of a sequence family shows the pattern of conservation i.e. some regions are more conserved by the family and some regions seem to tolerate insertions and deletions. The position specific information shows the degree of conservation in some positions and the degree of variation to which insertions and deletions are permitted. The Profile HMMs use this information for position specific scoring, e.g. there will be more penalty for insertion/deletion in a conserved region than a region of tolerance. While the traditional pairwise alignment, like BLAST [5] or Smith-Waterman [6], uses position independent scoring (i.e. gap penalties are globally fixed), thus the pattern of conservation in a sequence family are not considered. As the multiple sequence alignment concentrates on the features or key residues conserved by the family of sequences, it can find even a remote sequence homolog which can not be detected by a pairwise alignment. One of the most commonly used program for HMM analysis is the open source software suite HMMER, developed at Washington University, St. Louis by Sean Eddy [7.

HMMER involves very computationally demanding algorithms and accounts for a large amount of time spent in biological sequence analysis. Many authors have also investigated dedicated parallel hardware implementations, notably on FPGAs and GPUs. Recently, a new software implementation (HMMER3) has been launched and a great deal of effort has been put to improve its software execution through both fine grain (SIMD extension such as ALTIVEC and SSE extensions) and coarse grain parallelization (using MPI or multi-threads) [8]. Particularly, a DualCore SSE implementation of HMMER3 is faster than most of previous FPGA and GPU versions of HMMER2.

As currently defined and programmed, the latest version of HMMER spends 
most of its time in two kernel functions (called MSV and P7Viterbi). These two kernels contain loop carried dependencies (caused by the feedback path from the end to the beginning of model) which restricts any kind of parallelism.

We propose a technique to rewrite the computation in such a way that both kernels become very amenable to parallel implementation, while keeping all the original dependencies into account. Specifically, this paper makes two contributions. First, we describe how the original dynamic programming equations of MSV and P7Viterbi can be rewritten so as to develop the new algorithm that admits a scalable parallelization scheme at a price of a moderate, constant factor increase in the algorithm computational volume. Then we propose several strategies for efficiently implementing this improved algorithm ${ }^{1}$ on an FPGAbased High Performance Computing platform and discuss the performance that we obtained. For arbitrary profile sizes the performance degrades due to fixed size of design in hardware. We handle this issue by creating a library of preexisting configurations and loading the optimal configuration, at run time, for a given profile size.

This paper is organized as follows. Section 2 provides a detailed overview of previous work on parallelization of the HMMER software. Section 3 and 4 explain the approach we followed to rewrite the computations involved in the two kernels. Section 5 and 6 respectively focus on the FPGA implementation and on experimental results. Conclusion and future work directions are drawn in Section 7 .

\section{Background \& Related work}

\subsection{P7Viterbi Algorithm Description}

The P7Viterbi is the most time consuming kernel inside hmmsearch tool, which solves Plan7 HMMs through the well known Viterbi a dynamic programming algorithm. The architecture of Plan7 model is shown in Fig,1. The M

\footnotetext{
${ }^{1}$ In this work we are interested only in the hmmsearch software, as hmmpfam is known to be I/O bound
} 
(Matching), I (Insertion) and D (Deletion) states constitute the core section of the model. The states E, N, J, B and C are called "control states". The state $\mathrm{B}$ and $\mathrm{E}$ are dummy non-emitting states, representing the start and end of model, while state $\mathrm{N}, \mathrm{J}$ and $\mathrm{C}$ are used to control algorithm-dependent features like, local and multi-hit alignments. The states $\mathrm{S}$ and $\mathrm{T}$ are dummy states to represent start and termination of the process.

The states E, J and B form a feedback loop in the model(i.e., a cycle in the graph), which rarely changes the value of B and many hardware accelerators exploit this fact and ignore this feedback path, as will be shown in section 2.2

On the other hand, this feedback loop gives HMMER the ability to perform multiple hit alignments i.e. more than one segment per sequence can be aligned to the core section of model. The self-loop over J provides the separating sequence length between two aligned segments. Thus, from an algorithmic point 
of view, it is incorrect to ignore this edge in the model.

Algorithm 2.1: P7Viterbi( )

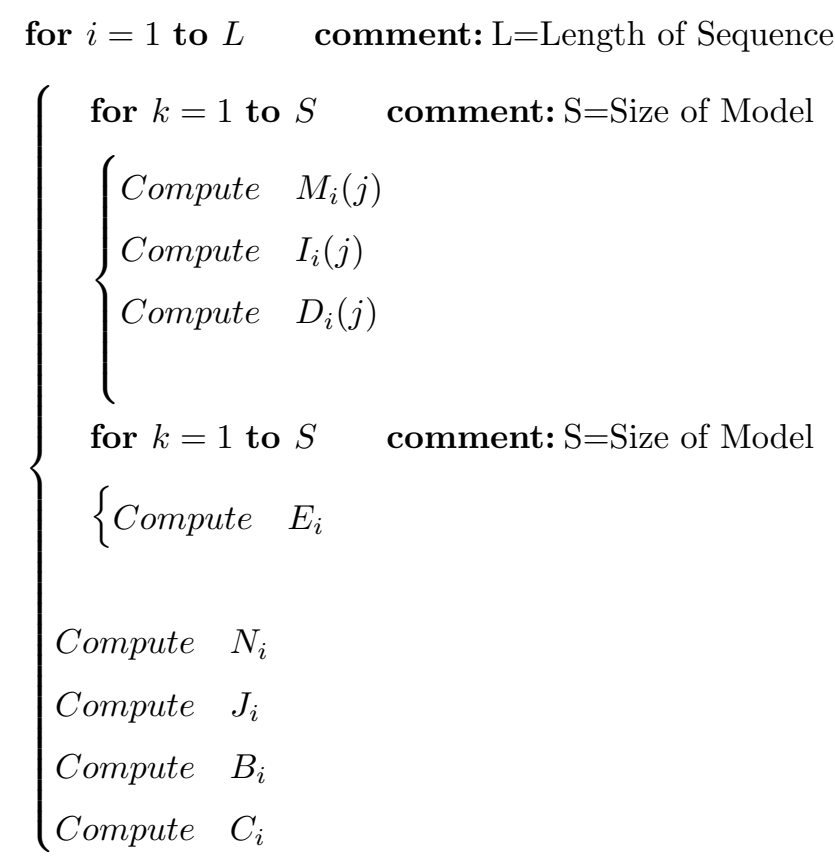




$$
\begin{aligned}
& M_{i}[k]=\max \left\{\begin{array}{l}
e_{M}\left(\mathrm{Seq}_{i}, k\right)+\max \left\{\begin{array}{l}
M_{i-1}[k-1]+\mathrm{TMM}[k] \\
I_{i-1}[k-1]+\mathrm{TIM}[k] \\
D_{i-1}[k-1]+\operatorname{TDM}[k] \\
B_{i-1}+\operatorname{trB}[k]
\end{array}\right.
\end{array}\right.
\end{aligned}
$$

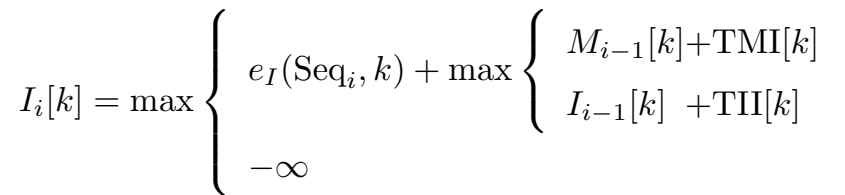

$$
\begin{aligned}
& D_{i}[k]=\max \left\{\begin{array}{l}
M_{i}[k-1]+\operatorname{TMD}[k] \\
D_{i}[k-1]+\operatorname{TDD}[k] \\
-\infty
\end{array}\right. \\
& E_{i}=\max \left\{\begin{array}{l}
M_{i}[k]+\operatorname{trM}[k] \\
-\infty
\end{array}\right. \\
& N_{i}=\max \left\{\begin{array}{l}
\left.N_{i-1}+\operatorname{trCnst}_{0}[N]\right) \\
-\infty
\end{array}\right. \\
& J_{i}=\max \left\{\begin{array}{l}
\left.E_{i}+\operatorname{trCnst}_{0}[E]\right) \\
J_{i-1}+\operatorname{trCnst}_{0}[J] \\
-\infty
\end{array}\right. \\
& B_{i}=\max \left\{\begin{array}{l}
\left.N_{i}+\operatorname{trCnst}_{1}[N]\right) \\
J_{i}+\operatorname{trCnst}_{1}[J] \\
-\infty
\end{array}\right. \\
& C_{i}=\max \left\{\begin{array}{l}
\left.C_{i-1}+\operatorname{trCnst}_{0}[C]\right) \\
E_{i}+\operatorname{trCnst}_{1}[E] \\
-\infty
\end{array}\right.
\end{aligned}
$$

The pseudocode for P7Viterbi can be seen in Algorithm 2.1, and Eq.(1,8) shows details for each computation.

The TXX, trB, trM, $e_{M}$ and $e_{I}$ are transition memories while trCnst is a set of constants. The Seq ${ }_{i}$ in Eq.(1) and Eq.(2) is the current sequence character 
being aligned.

The computations in (3) and (4) are most crucial while extracting parallelism. Early implementations used to ignore the (4) to simplify the computations. In our work, we rewrite the (3) such as the dependency of $D[i, j] \longrightarrow$ $D[i, j-1]$ is removed and all computations in column $j$ can be done in parallel. While computations in (4) can be done in a scalable manner.

In rest of the paper, we will express control states collectively as $X$, to emphasize the main part of the model.

\subsection{Early Implementations}

HMMER has received a lot of attention from the high performance computing community, with several implementations either for standard parallel machines or more heterogeneous architectures (9, 10, 11). In the following we will focus on hardware implementation targeting ASIC or FPGA technology.

Early proposals $([12,13,14,15])$ of hardware accelerator for profile based similarity search considered an (over) simplified version of the algorithm in which the feed-back loop is ignored as such a simplification happen to have a relatively limited impact on the actual quality of results of the algorithm.

The first hardware implementation of the exact algorithm was done by Oliver et al 16. In their work, they reconsidered the problem by taking advantage of the fact that in hmmsearch, multiple independent instances of the Viterbi algorithm are to be executed during each call. They used this fact to model the problem as a triple nested loop (instead of a simple double nested loop) in which the most outer loop is parallel and proposed a SIMD architecture for execution of the algorithm.

However, one of the issues with their parallelization scheme is that all processing elements need to concurrently access the (large) transition cost look-up tables. They addressed this problem by observing that all the processing element would only access a small subset of the table (24 locations, i.e., the number of bases in the amino acid alphabet). They therefore propose a vectorized memory with a 24-element wide data bus, in which each PE selects its cost value using 
a 24 to 1 multiplexer. Of course this approach suffers form severe scalability issues, which makes it impractical for massively parallel implementations.

Another approach was proposed by Derrien and Quinton [17]. It also uses the fact that many instances of the Viterbi algorithm can be processed in parallel, however the parallelization scheme (based on polyhedral space-time transformation) is more sophisticated than that of Oliver et al. [16] and allows one to derive a flexible architectural skeleton which does not need access to a shared memory for calculating the transition costs. The proposed approach also easily handles resource constraints by controlling the number of processing elements in the architecture, and allows for precise tuning of the datapath pipeline. Although the approach does not suffer from previous shortcoming, its scalability is still somewhat limited, as the local storage requirements of the hardware implementation can be prohibitive. For example a 64-element processing array with a 6 stage pipelined datapath, would need more than 500 embedded memory blocks on the FPGA.

\subsection{Speculative execution of the Viterbi algorithm}

More recently, an approach for hardware acceleration based on speculative execution was proposed by Takagi et al. [18] and Sun et al. [19]. Their idea is to take advantage of some property of the $\max$ operation, so as speculatively ignore the dependency over variable $B[i]$, (1), since it very seldom contributes to the actual computation of $M[i+1, k], D[i+1, k]$ and $I[i+1, k]$. This then results in a feedback-loop free algorithm, like [12]-13], which is very easy to parallelize.

However, whenever it is observed that the actual value of $B[i]$ would have contributed to the actual value of $M[i+1,0]$, all computations related to columns $i^{\prime}$ such as $i^{\prime}>i$ are discarded (flushed) and the computation must be re-executed so as to enforce the original algorithm dependencies. To do so Takagi et al. propose a misspeculation detection mechanism which stores in a buffer the values of $M, D$ and $I$ computed at the beginning of the new column (and their inputs) until the actual value of $B$ is available (that is $M_{\text {prof }}$ cycles later). The true 
values of $M, D$ and $I$ are then recomputed, and if they differ from the previous one, it means that the speculation was wrong, and that the previous results must be discarded.

One of the main issues with such an approach is the probability and cost of a misprediction. In this solution, whenever a misprediction occurs, the architecture has been running useless calculations during $M_{\text {prof }}$ cycles. Assuming a misspeculation probability $p$, the execution overhead for a sequence of $S$ amino acid bases can then be written as :

$$
e=\frac{S+M_{\text {prof }}}{S+M_{\text {prof }}+p S M_{\text {prof }}}
$$

As noticed by Takagi et al, the average observed value for $p$ is 0.0001 , which lead to an efficiency that vary between $94 \%$ and $99 \%$ depending on the depth of the speculation. It can also be observed that overhead is more important for an architecture exhibiting a large level of parallelism (the depth of the speculation being deeper), and for long sequences matched against small profiles, for which the probability of observing a repetition is cumulative with the sequence size. As an example Takagi et al. report cases where HMM profile characteristics lead to a poor efficiency (performance degradation by $85 \%$ ).

Very recently, Eusse Giraldo et al. 20] proposed another approach. They used simplified (without $J$ state) viterbi kernel as a filter and passes only sequences with significant scores to original viterbi kernel along with divergence algorithm 21] data. The divergence algorithm data reduces the number of cells that must be calculated with the original Viterbi kernel by providing limits of the alignment region. The alignment region defines where the alignment starts and ends. This approach yields an acceleration of 5.8 GCUPS (Giga Cell Updates Per Second).

However, the use of simplified Viterbi as a filter may not detect multiple hit alignments. As the filter also specify the alignment region to the original Viterbi kernel the original kernel will not try to align segments of the sequence lying outside the alignment region, which may produce falsenegatives. The paper 
doesn't discuss issues with multiple hit alignments, and how this is handled inside simplified Viterbi filter.

\subsection{GPU Implementations of HMMER}

The HMMER search was implemented on graphics processing units by Horn et al. 22. as Claw-HMMER and later by Walter et al. [9. The overall speed up reported by Walter is 15 to $35 \times$ on a single NVIDIA 8800 GTX Ultra GPU in comparison with software implementation. The GPU implementations lack very high speedup due to extensive global memory access by P7Viterbi algorithm.

A very recent implementation on GPU by Ganesan et al. 23] accelerated the HMMER by breaking the chain of dependencies inside P7Viterbi. The reported speed up is $100+$ times on 4 Tesla C1060 GPUs in comparison with software implementation of HMMER2. They converted the vertical cell dependency $D[i, k] \longrightarrow D[i, k-1]$ into dependencies between equal sized chunks of a column. In first step, the headers of chunks are updated serially, then the intermediate values of each chunk can be computed in parallel. They followed almost similar strategy as we do for breaking this dependency in computation of $D$, but we convert this dependency into well-known parallel prefix networks. The parallel prefix network topologies provide the freedom to compromise between delay and area cost according to the architecture requirement, as shown in later sections.

\subsection{HMMER3 and The Multi ungapped Segment heuristic}

The new version of HMMER, which is available for use now, is a radical rewrite of the original algorithm, with a clear emphasis on speedup. The most noticeable difference in this new version lies in a new filtering heuristic (called Multi ungapped Segment Viterbi) which serves as a prefiltering step, and is executed before the standard P7Viterbi in the HMMER pipeline as illustrated in Fig. 2. This algorithmic modification alone helps improve speedup by a factor of 10, and its algorithm is outlined in Algorithm 2.2. 
It is important to note that the MSV still holds the feedback loop $(E \rightarrow J \rightarrow$ $B)$, which restricts to start $M(i, j+1)$ until $M(i, j)$ is finished. But as compared to algorithm 2.1. the computations of $D(i, j)$ and $I(i, j)$ are removed and hence $M(i, j)$ no longer depends on these computations, which gives opportunity for faster computation.

Algorithm 2.2: MSV( )

for $i=1$ to $L \quad$ comment: $\mathrm{L}=$ Length of Sequence

$$
\left\{\begin{array}{l}
\text { for } k=1 \text { to } S \quad \text { comment: } \mathrm{S}=\text { Size of Model } \\
\left\{\begin{array}{l}
\text { Compute } M^{\prime}(i, j) \\
\text { Update } E(i)
\end{array}\right. \\
\text { Compute } N(i) \\
\text { Compute } J(i) \\
\text { Compute } B(i) \\
\text { Compute } C(i)
\end{array}\right.
$$

In addition to this filtering step, both P7Viterbi and MSV algorithm have also been redesigned so as to operate on short wordlengths ( 8 bits for MSV and 16 bits for P7Viterbi) so as to fully benefit from the SIMD extensions (SSE, Altivec) available on all Intel/AMD CPUs. The new HMMER3 is claimed to run at about BLAST speed; slightly faster than WU-BLAST and somewhat slower than NCBI BLAST [24].

As shown in table 1, the combination of the MSV pre-filtering stage with SIMD has a huge impact of the overall software performance, which is improved by a factor of more than 100, and which actually makes most previous FPGA implementations acceleration slower than any recent Dual-core CPU machine, as shown by Table 2 . 


\subsection{Accelerating the complete HMMER3 pipeline}

As shown in Fig. 2, because the MSV algorithm is used as a prefiltering step, the P7Viterbi algorithm still contributes in a non-negligible way to the execution time. In other words, significantly improving global execution time cannot be done by only accelerating the MSV kernel alone, and there is still a need for efficiently accelerating the P7Viterbi algorithm.

In the following section we propose to rewrite both MSV and P7Viterbi algorithms to make them amenable to hardware acceleration. We do so by using a simple reformulation of MSV equations to expose reductions operations, and by using an adaptation of the technique proposed by Gautam and Rajopadhye 25] to detect scans and prefix computations in P7Viterbi. This exposes a new level of parallelism in both algorithms that was previously unknown.

\section{Rewriting the MSV Kernel}

As mentioned earlier, the main computation in the MSV kernel is a dynamic programming algorithm that follows the standard algorithmic technique of filling up one data tables (called $\mathrm{M}[\mathrm{i}, \mathrm{k}]$ in this paper with $\mathrm{i}$ as the column index, and $\mathrm{k}$ as the row index) together with some other auxiliary variables. The values of the table entries are determined from previously computed entries (with appropriate initializations) using the following formulas.

$$
\begin{aligned}
M[i, k] & =\operatorname{MSC}[k]+\max \left(M[i-1, k-1], X_{b}[i-1]\right) \\
X_{e}[i] & =\max _{k}(M[i, k]) \\
X_{n}[i] & =\max \left(X_{n}[i-1]+\text { tloop, }-\infty\right) \\
X_{b}[i] & =\max \left(X_{n}[i], X_{e}[i], X_{e}[i-1]\right)
\end{aligned}
$$

It can be observed that the computation of $M_{i}$ has a diagonal dependency for column $M_{i-1}$ and $X_{b}$, where $X_{b}$ depends on all value of $M_{i-1}$ i.e. any computation for column $M_{i}$ can not start, until the all the computations for the column $M_{i-1}$ are computed, which gives column-wise sequential execution to the algorithm. 
On the other hand, all values of a given column $M_{i}$ can be computed in parallel. Since the computation of $X_{e}$ consists of a max reduction operation, this can be realized using a max tree computation, as shown in Fig 3 , reducing latency of MSV architecture from $\mathrm{O}(N)$ to $\mathrm{O}\left(\log _{2} N\right)$.

\section{Rewriting the P7Viterbi Kernel}

As shown in previous section, it is easy to rewrite the MSV algorithm recurrence equations so as to expose parallelism in the form of a simple max-reduction operation.

In this Section, we show how it is also possible to use a similar (but more complex) rewrite on the P7Viterbi kernel. Here again, the goal is to get rid of the current inherent sequential behavior caused by the so-called feed-back loop. To do so, we replace the accumulation along the $j$ index for one of the variables by a prefix-scan operation and replace the feed-back loop by a simple max-reduction operation. This transformation leads to a modified dependence graph which is much better suited for a parallel hardware implementation.

$$
\begin{aligned}
M_{i}[k] & =f_{M}\left(M_{i-1}[k-1], I_{i-1}[k-1], D_{i-1}[k-1], X_{i-1}\right) \\
I_{i}[k] & =f_{I}\left(M_{i-1}[k], I_{i-1}[k]\right) \\
D_{i}[k] & =f_{D}\left(M_{i}[k-1], D_{i}[k-1]\right) \\
X_{i} & =f_{X}\left(\max _{k}\left(M_{i}[k]+E[k]\right)\right)
\end{aligned}
$$

The key observation concerning P7Viterbi formulas $(13,16)$ is that

- there is a chain of dependences in the increasing order of $k$ in computing the values of $D$ in any column;

- to compute the $X$ for any column, we need all the values of $M$ of that column, each of which needs a $D$ from the previous column; and

- the value of $X$ of a column is needed to compute any $M$ in the next column.

Because of this, there seems to be an inherent sequentially to the algorithm, as noted by all previous work on this problem. 


\subsection{Finding reductions}

We now develop an alternate formulation of the equations so that there is no such chain of dependences, thus enabling scalable parallelization of the computations on a hardware accelerator.

More specifically, we show that the equation computing $D$ can be replaced by a different equation in which such dependences either do not exist, or can be broken through well-known techniques. For our purposes, we shall focus on the function $f_{D}$, which is defined more precisely as follows:

$$
D_{i}[k]=\left\{\begin{array}{lll}
k=1 & : & M_{i-1}[0]+A^{\prime}[0] \\
k>1 & : & \max \left(D_{i}[k-1]+B[k], M_{i-1}[k]+A^{\prime}[k]\right)
\end{array}\right.
$$

where $A^{\prime}$ is TMD, and $B$ is TDD memories, reffering to eq. 3 . From the point of view of the computation in the $i$-th column, we can consider the values for the previous column (viz., the $M_{i-1}$ terms above) as inputs, and so the equation can be further abstracted as follows:

$$
D_{i}[k]=\left\{\begin{array}{lll}
k=1 & : & a_{0} \\
k>1 & : & \max \left(D_{i}[k-1]+b_{k-1}, a_{k-1}\right)
\end{array}\right.
$$

Now, if $B$ is zero, the equation is a simple scan computation (also called prefix computations) $D_{i}[k]=\underset{i=1}{k} a_{i}$.

It is well known how to efficiently and scalably parallelize such scan computations [26. However, if $B \neq 0$, the solution is not at all obvious. We show below how to obtain a scan-like structure for this case. If we expand out the 
individual terms, we see that:

$$
\begin{aligned}
D[1]= & a_{0} \\
D[2]= & \max \left(a_{0}+b_{1}, a_{1}\right) \\
D[3]= & \left.\max \left(\max \left(a_{0}+b_{1}, a_{1}\right)+b_{2}, a_{2}\right)\right) \\
= & \max \left(a_{0}+b_{1}+b_{2}, a_{1}+b_{2}, a_{2}\right) \\
D[4]= & \max \left(a_{0}+b_{1}+b_{2}+b_{3}, a_{1}+b_{2}+b_{3}, a_{2}+b_{3}, a_{3}\right) \\
\vdots & \\
D[k]= & \max \left(a_{0}+b_{1}+b_{2}+b_{3} \ldots b_{k-1}, a_{1}+b_{2}+b_{3} \ldots b_{k-1},\right. \\
& \left.a_{2}+b_{3} \ldots b_{k-1}, \ldots a_{k-2}+b_{k-1}, a_{k-1}\right)
\end{aligned}
$$

The last term can be written more visually as

$$
D[k]=\max \left(a_{k-1}, \max \left(\left(\begin{array}{r}
b_{1}+b_{2}+b_{3}+\ldots+b_{k-1} \\
b_{2}+b_{3}+\ldots+b_{k-1} \\
b_{3}+\ldots+b_{k-1} \\
\vdots \\
\\
b_{k-1}
\end{array}\right)+\left(\begin{array}{c}
a_{0} \\
a_{1} \\
a_{2} \\
\vdots \\
a_{k-2}
\end{array}\right)\right)\right)
$$

Writing this more compactly, we proceed as follows.

$$
D[k]=\max \left(a_{k-1}, \max _{j=1}^{k-1}\left(a_{j-1}+\sum_{i=j}^{k-1} b_{i}\right)\right)
$$

In above expression (19), one can easily identify the reduction operation over vector $b$ and max-prefix over this reduction operation. But still reduction operation depends on inner loop index and we would like to get rid of it. To accomplish this job, we add and subtract same term which will not effect the computation and will yield following expression:

$$
D[k]=\max \left(a_{k-1}, \max _{j=1}^{k-1}\left(a_{j-1}+\sum_{i=j}^{k-1} b_{i}+\sum_{i=1}^{j-1} b_{i}-\sum_{i=1}^{j-1} b_{i}\right)\right)
$$


The term $\sum_{i=1}^{k-1} b_{i}=\sum_{i=j}^{k-1} b_{i}+\sum_{i=1}^{j-1} b_{i}$ is independent of $j$, so it can be moved out.

$$
D[k]=\max \left(a_{k-1},\left(\sum_{i=1}^{k-1} b_{i}+\max _{j=1}^{k-1}\left(a_{j-1}-\sum_{i=1}^{j-1} b_{i}\right)\right)\right)
$$

Now note that $\sum_{i=1}^{j-1} b_{i}\left(\right.$ say, $\left.b_{j-1}^{\prime}\right)$ is a scan of the $b$ input, so

$$
\begin{aligned}
D[k] & =\max \left(a_{k-1}, b_{k-1}^{\prime}+\max _{j=1}^{i-1}\left(a_{j-1}-b_{j-1}^{\prime}\right)\right) \\
& =\max \left(a_{k-1}, b_{k-1}^{\prime}+\max _{j=1}^{i-1} a_{j-1}^{\prime}\right)
\end{aligned}
$$

where $a_{j}^{\prime}=a_{j}-b_{j}^{\prime}$ is the element wise difference of $a$ and $b^{\prime}$.

Now, the inner term is max-scan of the $a^{\prime}$ vector. Hence the $D[k]$ as specified by Eqn. 21 can be computed in parallel using the following steps.

1. Compute, $b^{\prime}$ the sum-prefix of the array $b$. Note that in the Viterbi algorithm, this needs to be done only once since $B$ is an input.

2. Compute $C$, the element wise subtraction of this from $A$.

3. Perform a max-prefix on $C$. This can be parallelized perfectly and scalably.

4. Add $b^{\prime}$ element wise to this and compare (again element wise) the result with the $A$ input, retaining the larger one. This yields $D$, the desired answer.

We have rewritten the dependency $D[i, j] \longrightarrow D[i, j-1]$, and now vector $D$ can be computed in parallel by following above steps, where computation path is converted into a max-prefix network rather than a strict intra-column dependency.

\subsection{Impact of the data-dependence graph}

To help the reader understanding the benefits of this rewriting transformation, we provide in Fig. 4 an illustration of the data dependence flow in the rewritten algorithm for a small problem size (profile size $N=8$ ). In this 
dataflow graph, functions $f_{i, k}, g_{i, k}$ and $h_{i, k}$ are defined as follows, referred to eq. 1] 3 :

$$
\begin{aligned}
f_{M}(w, M, D, I) & =\max _{4}\left(w+\operatorname{tr} B_{k}, M+T M M_{k}, D+T D M_{k}, I+T I M_{k}\right)+e M_{k}\left[S e q_{i}\right] \\
g_{I}(M, I) & =\max _{2}\left(M+T I I_{k}, I+T M I_{k}\right)+e I_{k}\left[S e q_{i}\right] \\
h_{D}(M, D) & =\max _{2}\left(M+T M D_{k}, D+T D D_{k}\right)
\end{aligned}
$$

In these expressions max and sum correspond to saturated (w.r.t to $-\infty$ ) max and sum operations. It can be observed that there is no longer a chain of dependencies along the vertical axis in the data-flow graph, and that the longest path (i.e. critical path) is now set by the depths of the parallel max-tree and the parallel max-prefix blocks, which is $O\left(\log _{2}(N)\right)$. Another consequence is that update operations for $M_{i, k}, I_{i, k}$ and $D_{i, k}$ can be executed in parallel for all values of $k$ in the domain $0 \leq k \leq N$.

\section{Hardware Mapping}

Even though the rewritten version of both MSV and P7Viterbi algorithms exhibits a significant amount of hidden parallelism, deriving a efficient architecture from the modified dataflow graph is not straightforward.

In this section we address the different challenges involved in this architectural mapping. We first start by discussing efficient hardware implementation of parallel prefix operations as needed by P7Viterbi, and present two transformations (namely C-Slow and tiling) that we use to improve the architecture efficiency.

\subsection{Implementing the max-prefix operator}

As mentioned in Section 4.1, step 3, the rewritten version of the P7Viterbi algorithm exhibits a max-prefix pattern. Prefix computation is a very general class of computations which can be formally defined as follows : given an input vector $x_{i}$ with $0 \leq i<N$ we define its $\oplus$-prefix vector $y_{i}$ as : 


$$
y_{i}=\bigoplus_{k=0}^{i} x_{k}=x_{0} \oplus x_{1} \oplus \ldots \oplus x_{i}
$$

Where $\oplus$ is a binary operator with associativity (and possibly commutativity, see [27] for a more detailed definition). Because binary adders fall into this category and since adders form one of the most important building blocks in digital circuits, there is a wealth of research going back almost 50 years dealing with fast (i.e parallel) implementations of prefix adders [26, 28, 29, 30, 31] using various interconnection networks topologies.

One of the most important aspects of these network topologies is that they allow the designer to explore the trade-off between speed (i.e. critical path of the resulting circuit), area (number of operators used to implement the prefix operation), and other metrics such as fan-out or wiring length. A comprehensive classification, describing the trade-offs in existing network topologies, has been done in 32 .

For example, Fig. 5]a shows a Brent-Kung [28] network that computes the prefix in $2\left(\log _{2} N-1\right)$ stages with $2(N-1)-\log _{2} N$ operators. Similarly, Fig. 5.b shows a Sklansky network which implements a faster circuit $\left(\log _{2} N\right.$ stages $)$ at a price of an increase in area $\left(\begin{array}{c}N \\ 2\end{array} \log _{2} N\right.$ operators $)$.

Although a survey of all existing techniques and network topologies is out of the scope of this paper, we provide in table 3 a short summary of the characteristics of the most commonly used algorithms. Note that most of these algorithmic explorations were in a context where the operator was extremely fine grain - just a few Boolean gates, as in a half- or full-adder.

Despite the fact that our computation scheme is based on the same prefix patterns as binary adders, our situation differs in two ways :

- The basic operation is not a bit-level (i.e full-adder) but a more complex word level operation (namely max).

- The size of the prefix can be very large (up to 256 input elements) which poses scalability issues in terms of routing. 
To the best of our knowledge there has been no systematic study of FPGA implementation of prefix computations. One reason is that the typical use of such circuits would be in adders, where high-speed carry circuits are already provided by FPGA vendors, and there are few applications that need coarsegrain, word-level operators. For the HMMER application we implemented a number of the max-prefix as well as max-reduce architectures. The performance comparisons are reported later in Section 6.2

\subsection{Architecture with a single combinational datapath}

It can be easily seen from Fig, 4 and Fig 3 , that in both MSV and P7Viterbi, it is not possible to pipeline the execution of consecutive stages - all the results of the $i^{\text {th }}$ stage are needed before ANY value in the $(i+1)^{t h}$ stage can be computed.

As a consequence, and in spite of the fact that we replaced in both cases the initial chain of dependence of $O(M)$ operations by a chain of $O\left(\log _{2}(M)\right)$, the possibly large values of $M$ may induce a large critical path, which could lead to a poor clock frequency.

\subsection{A C-slowed pipelined datapath}

To obtain a datapath with better clock frequency, pipelining is always an adequate choice. Pipelining data paths without feedback loop results in fast and efficient designs. However pipelining becomes ineffective when there is a feedback loop. One can observe from the Fig 6(a) that the pipeline is never filled completely, due to dependence of the logic block 'A' over results of the logic block 'C'. The new sample will wait until the results of previous sample are calculated, hence only one logic block will be active at any clock cycle.

As HMMER hardware will be always run for a set of sequences, which are independent of each other, so a wise choice is to input interleaved multiple sequences to the dataflow. The resulting architecture is shown in Fig 6(b). This method will cost extra registers i.e. (C-Slow $\times$ pipeline stages). But in return, our architecture will be as effecient as a normal pipelined architecture without a feedback loop. 
Same solution to improve throughput of the hardware implementation has been used by Derrien and Quinton [17, and also Oliver et al. [16] by applying a C-slow transformation on the generated datapath so as to interleave several executions of the dataflow graph (i.e., several independent instances of the MSV/P7Viterbi algorithm).

Another way to view this transformation is to consider that we add an additional outer loop iterating over independent instances of the algorithm, and then perform a loop interchange so as to move this parallel outer loop to the innermost level and implement the multiple independent instances on pipelined hardware in parallel.

Using this trick, and assuming the interleaving of $S$ independent instances, the $i^{\text {th }}$ stage now only depends on stage that was executed $i-S$ stages ago. This extra delay can then be used to pipeline the stage execution, as depicted in Fig. $7(\mathrm{a})$,

This of course has some additional memory cost (we must replicate all registers/memories in the architecture), but because the critical path remains in $O\left(\log _{2} M\right)$, we only need a reasonably small C-slow factor to achieve the maximum throughput (as compared to $S \approx O(M)$ in the approach of Derrien and Quinton).

\subsection{Managing resource constraints through tiling}

Both MSV and P7Viterbi dataflow graphs sizes scale linearly ${ }_{1}^{1}$ with the target HMM profile size $M$. For large values of profile sizes ( e.g., greater than 100) the straightforward mapping of the complete dataflow graph to a hardware datapath quickly becomes out of reach of most FPGA platforms.

However, since the computational pattern of both algorithms exhibits a lot of regularity, it is possible to apply a simple tiling transformation, which tiles each dataflow of size $M$ into $P$ partitions, each of them calculating $M / P$ con-

\footnotetext{
${ }^{1}$ The scaling is linear for the Brent-Kung architecture that we implemented. For the Ladner-Fischer architecture, the resource usage grows as $n \log n$
} 
secutive values of the current column. This transformation, and its impact on the scheduling of computation is depicted in Fig. 7(b) In the case of MSV, the partitioned datapath should implement a $M / P$ reduction max operator, whereas in the case of P7Viterbi, we need a $M / P$ max prefix operation.

As a summary, the characteristics of various design space points that we explored are listed in Table 4

\subsection{Accelerating the full HMMER pipeline}

As mentioned in subsection 2.6, improving global performance requires that both MSV and P7Viterbi are accelerated in hardware. This can be done by streaming the output of MSV to the input of the P7Viterbi, so as to map the complete HMMER3 pipeline to hardware. Special care must be given to C-Slow factor for both accelerators, which must be the same so as to avoid a complex data reorganization engine between the two accelerators.

In addition, depending on available resource, it is even possible to instantiate several HMMER3 pipelines in parallel, as illustrated in Fig. 8. To implement C-Slow, sequences are interleaved on hardware. The "Sequence DEMUX" receives serialized sequences and produces C-Slow interleaved sequences to each MSV and P7Viterbi block. Similarly, "Sequence MUX" receives filtered sequences from filter and produces serialized form to the DEMUX. Fig. 9 shows the sequence handling inbetween MSV blocks and P7Viterbi block.

However, in order to optimize hardware resource usage, we must also ensure that the pipeline workload is well distributed among the hardware accelerators. Let us quantify the total algorithm execution time, written as $T_{\text {total }}$ when the two task executions are pipelined, we have :

$$
T_{\text {total }}=\max \left(T_{\mathrm{msv}}, \alpha T_{\text {viterbi }}\right)
$$

Where $T_{M S V}$ and $T_{V i t e r b i}$ correspond to the average algorithm execution times, and where $\alpha$ is the filtering selectivity. Optimizing performance therefore means ensuring the raw performance (in GCUPS) of the P7Viterbi accelerator 
is able to sustain the filtered output of the MSV accelerator, that is, its performance should be at least $1 / 50^{t h}$ that of MSV, Fig. 2. Using this constraint we can then define a set of pipeline configurations, by choosing distinct tiling parameters (i.e. partition sizes) for P7Viterbi and MSV such that the level of parallelism exposed in MSV is at least $50 \times$ that of P7Viterbi.

\section{Experimental results}

In this section we provide an in-depth quantitative analysis of our proposed architectures, and compare their performance with that of state of the art software implementation of HMMER3 on CPU using the SSE SIMD implementation.

Our target execution platform consists of a high-end FPGA accelerator from XtremeData (XD2000i-FSBFPGA) which has already been successfully used for implementing bioinformatics algorithms [33. This platform contains two Stratix-III 260 FPGAs, high bandwidth local memory (8.5 GBytes/s) and a tight coupling to the host front side bus though Intel Quick Assist technology, providing sustained 2 GBytes/s bandwidth between the FPGA and the host main system memory.

Our design flow leverages high-level synthesis through a commercial C to Hardware compiler (Impulse CoDeveloper C-to-FPGA) combined with the Gecos 34 framework, a semi automated source to source compiler targeted at program transformations for High Level Synthesis. The combined use of these two tools allowed us to explore a very large architectural design space in a very reasonable amount of time.

The rest of the section is organized as follows: we first make a quantitative analysis of speed/area results for the MSV accelerator, then address the mapping of max prefix network implemented on FPGA along with P7Viterbi implementation results. Finally, we discuss system level performance and compare performance of our approach with that of an hypothetical, state of the art GPU implementation. 


\subsection{Area/Speed results for the MSV filter}

Table 5 summarizes area and speed results obtained for several MSV hardware accelerators using different values of $M$ and $S$ (the MSV accelerator doesn't need tiling as for all profile sizes, it fits in the FPGA). It can be observed, that even though we use a $\mathrm{C}$ to hardware high level synthesis tool, we are able to achieve remarkably high operating frequencies (up to $215 \mathrm{MHz}$ ). When compared 2 with that of table 1 results indicate speedup for a single accelerator varying between $3 \times$ to $6 \times$ depending on $M$.

\subsection{Area/Speed results for max-prefix networks}

As mentioned in Section5, the P7Viterbi implementation uses a parallel max prefix scheme, for which many implementation schemes exist. As this computational pattern is at the core of the modified algorithm, we explored several alternative implementations so as to experimentally quantify their respective merits with respect to an FPGA implementation. We used an in-house Java based RTL generator, to generate these network topologies. In Java, a network topology is described in the form of loops and recursive function calls. A similar but more generalized approach for prefix network generation from abstract specification has been presented in [35], by modeling prefix networks using functional programming language, Haskell. In our case, the network construction in Java is more practical, as the objective is solely to generate RTL description for existing prefix networks and evaluate their performance on FPGA.

The results provided in Table 6 and Figure 10 show that for large values of $M$, so called fast implementations of parallel prefix such as Kogge-Stone or Ladner-Fischer provide only marginal speed improvements with respect to the Brent-Kung architecture. This can easily be explained by the long wires used in the first two approaches, which make the routing much more challenging

\footnotetext{
${ }^{2}$ This is an rough approximation, as we should also account for the time spent by the software in P7Viterbi (20\% of the total execution time)
} 
on an FPGA. We therefore decided to use Brent-Kung architecture for our implementation.

\subsection{Area/Speed results for the P7Viterbi filter}

Table 7 summarizes area and speed results obtained for several P7Viterbi hardware accelerators using different values of $M, S$ and $P$. It can be observed, that re-written P7Viterbi kernel can deliver quite promising speed with $\log _{2}(M)$ CSlow factor. By fitting multiple instances of P7Viterbi on board, It can alone(i.e. not using MSV filter) perform better than earlier implementation of HMMER2.

\subsection{System level performance}

So far, we have provided area/performance results only for standalone accelerator modules, which should be integrated together in one or several complete HMMER3 computation pipelines.

Following the constraints on pipeline workload balancing, and given the resources available implementing the accelerator on the board, we derived a set of pipeline configurations depending on the target profile size $M$.

These configurations have parameters (C-Slow factor $S$, Tiling parameter $P)$ chosen so as to maximize overall performance. The set of parameters for a given value of $M$ is chosen as follows:

- First, we choose the C-slow factor $S$ so as to enable the fine grain pipelining (i.e. at the operator level) of the MSV accelerator. The same value is then used for the pipelining the P7Viterbi accelerator.

- Second, we choose the Partition $P$ size so that the P7Viterbi accelerator can sustain with the MSV input through-put.

Table 8 describes some Quartus P\&R data for the pipeline configurations that we derived through this approach. The results presented correspond to a single pipeline implementation, i.e. a single MSV module followed by a single P7Viterbi moduele. These results show that speedups of up to $6.5 \times$ can be 
achieved for a single pipeline implemented on one out of the two FPGAs of the platform.

To implement multiple HMMER3 pipelines, we followed two approaches :

- First, a typical approach will be to keep all pipelines independent of each other and connect each MSV block to its own P7Viterbi block. At the end, results will be collected from all P7Viterbi blocks.

- Second, a better way, to utilize both available hardware resources and HMMER filtering characteristics will be to couple a greater number of MSV blocks to a smaller number of P7Viterbi blocks (as suggested in Fig. 88.

By implementing multiple HMMER3 pipelines, using first approach, we should be able to also improve the overall speed for smaller profile sizes (e.g. 64 and 128). Indeed, our estimates show that $7 \times$ speedups could be achieved compared to our baseline DualCore SSE implementation. Similarly, by using both FPGAs on board, we could double the number of HMMER3 pipelines running on the XD2000i platform. However due to firmware and device driver limitations, only one of the two FPGAs can be used at a time.

The first approach lacks load balancing after the MSV filters which might result in few busy and few idle P7Viterbi units. However, in the second approach the Sequence DEMUX units work as load balancing components by collecting the sequences and distributing them evenly between computation components. While selecting the number of MSV and P7Viterbi components, the Eq. 22 should be in consideration to keep the $T_{M S V}$ and the $T_{V i t e r b i}$ in balance.

Table 9 shows the $\mathrm{P} \& \mathrm{R}$ results for system level view described in Fig.88. It can be seen that by fitting multiple HMMER3 pipelines, a speedup of $\approx 7.4 \times$ can be achieved compared to DualCore SSE implementation. As profile size $M$ varies between 50 to 650 [36, the design should be able to handle arbitrary size of $M$ and sustain its speedup. This can be managed by taking advantage of the reconfigurable nature of FPGAs. For a given profile size, we can choose and 
load the configuration, at run time, which best suits that specific profile size from a set of predetermined bitstream configurations.

\subsection{Discussion}

One question raised by our results is whether implementation of complex system level architecture, as shown in Fig. 8 , is beneficial or not. The area cost for auxiliary components(e.g. MUX, DEMUX, Sequence Interleaver) becomes much higher than area cost for such components in independent pipelines. Although the architecture allows to implement fewer P7Vitervi components and conserve resources, but still the increased area cost for these additional components restricts the overall speedup improvement in comparison with independent pipelines. It can be concluded that while implementing complex architecture on hardware, a high implementation effort may result in a marginal speedup compared to a simple architecture involving fewer auxiliary components.

Another important point of discussion can be whether our FPGA would actually perform faster than an equivalent GPU implementation. This is an important point as GPU offers more flexibility at a much lower cost than a typical HPC FPGA platform, albeit at a higher cost in power/energy consumption. Unfortunately, there is currently no GPU version of HMMER3 available for comparing the two targets.

We however believe that, contrary to HMMER2, a GPU version of HMMER3 would only offer marginal speedup w.r.t the optimized SSE version. The GPU speedup for HMMER2 were reported in the order of $15-35 \times 9$ and $20-70 \times$ 23] for a single GPU over the software implementation. While the HMMER3 gives $230 \times$ performance improvement compared to that baseline.

When looking at HMMER3 speedup results (given in Table 1), it turns out that the use of an optimized SSE software implementation alone brings up to $20 \times$ speedup improvement over the non SSE version, a speed-up somewhat comparable to the GPUs implementation for HMMER2, and which is mostly due to the systematic use of sub-word parallelism. 


\section{Conclusion}

In this paper, we have proposed a hardware accelerator architecture for the new HMMER3 profile based search software. This architecture leverages on a rewriting of the algorithm to expose reduction and prefix scan computation patterns without modifying the semantic of the original algorithm. This rewriting permit a full and efficient parallelization of the two kernels on hardware, and is combined with an architectural design space exploration stage so as to determine the best performing architecture for a given HMMER profile size, by taking into account the amount of available hardware resource and the pipeline workload balance.

An improvement would be to take advantage of data reusability. Because HMMER is often used in a context where a profile database is matched against a sequence database, reducing the bandwidth pressure by using the accelerator on-board memory to store part of the input data-set seems an attractive option. The sequence database can be received in the beginning and stored in on-board memory and it can be utilized for each profile in HMM profile database. Indeed, it can be observed that, for small HMM profiles (say $M<128$ ), the combined through-put of a complete system level accelerator (with several pipelines) gets very close to the maximum through-put supported by the board.

\section{Acknowledgment}

This work has been supported by the French ANR BioWIC (ENR-08-SEGI005).

The work of Rajopadhye was supported by the National Science Foundation under awards No CCF-0917319 and 0811852

\section{References}

[1] A. Krogh, M. Brown, I. S. Mian, K. Sjölander, D. Haussler, Hidden Markov Models in Computational Biology: Applications to Protein Modeling, Journal Molecular Biology 235 (1994) 1501-1531. 
[2] S. R. Eddy, Profile hidden markov models, Bioinformatics 14 (9) (1998) $755-763$.

[3] D. Haussler, A. Krogh, I. Mian, K. Sjolander, Protein modeling using hidden markov models: analysis of globins, in: Proceeding of the Twenty-Sixth Hawaii International Conference on System Sciences, Vol. i, 1993, pp. 792 802 vol.1. doi:10.1109/HICSS.1993.270611.

[4] T. Jaakkola, D. Haussler, Exploiting generative models in discriminative classifiers, in: Advances in Neural Information Processing Systems 11, MIT Press, 1998, pp. 487-493.

[5] S. Altschul, T. Madden, A. Schffer, J. Zhang, Z. Zhang, W. Miller, D. Lipman, Gapped BLAST and PSI-BLAST: a New Generation of Protein Database Search Programs., Nucleic Acids Research (1997) 3899-3402.

[6] T. F. Smith, M. S. Waterman, Identification of common molecular subsequences. Journal of molecular biology 147 (1) (1981) 195-197.

URL http://view.ncbi.nlm.nih.gov/pubmed/7265238

[7] S. Eddy, HMMER3: a new generation of sequence homology search software, http://hmmer.janelia.org/.

[8] S. R. Eddy, Accelerated profile HMM searches (preprint) (2011).

[9] J. P. Walters, V. Balu, S. Kompalli, V. Chaudhary, Evaluating the use of gpus in liver image segmentation and hmmer database searches, in: IPDPS '09: Proceedings of the 2009 IEEE International Symposium on Parallel\&Distributed Processing, IEEE Computer Society, Washington, DC, USA, 2009, pp. 1-12. doi:http://dx.doi.org/10.1109/IPDPS.2009. 5161073

[10] B. Wun, J. Buhler, P. Crowley, Exploiting Coarse-Grained Parallelism to Accelerate Protein Motif Finding with a Network Processor, in: PACT '05: Proceedings of the 14th International Conference on Parallel Architectures and Compilation Techniques, 2005. 
[11] J. Lu, M. Perrone, K. Albayraktaroglu, M. Franklin, HMMer-Cell: High performance protein profile searching on the cell/b.e. processor, in: IEEE International Symposium on Performance Analysis of Systems and Software, 2008, pp. 223 -232. doi:10.1109/ISPASS.2008.4510754.

[12] R. P. Maddimsetty, J. Buhler, R. D. Chamberlain, M. A. Franklin, B. Harris, Accelerator Design for Protein Sequence HMM Search, in: Proceedings of the ACM International Conference on Supercomputing, ACM, Cairns, Australia, 2006.

[13] T. Oliver, B. Schmidt, Y. Jakop, D. L. Maskell, Accelerating the Viterbi Algorithm for Profile Hidden Markov Models Using Reconfigurable Hardware., in: International Conference on Computational Science, 2006.

[14] K. Benkrid, P. Velentzas, S. Kasap, A high performance reconfigurable core for motif searching using profile hmm, in: NASA/ESA Conference on Adaptive Hardware and Systems, 2008, pp. 285 -292. doi:10.1109/AHS. 2008.16

[15] A. C. Jacob, J. M. Lancaster, J. D. Buhler, R. D. Chamberlain, Preliminary results in accelerating profile hmm search on fpgas, in: Workshop on High Performance Computational Biology (HiCOMB), 2007.

[16] T. Oliver, L. Y. Yeow, B. Schmidt, High Performance Database Searching with HMMer on FPGAs, in: HiCOMB 2007, Sixth IEEE International Workshop on High Performance Computational Biology, 2007.

[17] S. Derrien, P. Quinton, Parallelizing HMMER for hardware acceleration on FPGAs, in: ASAP 2007, 18th IEEE International Conference on Application-specific Systems, Architectures and Processors, 2007.

[18] T. Takagi, T. Maruyama, Accelerating HMMER Search Using FPGA, in: International Conference on Field Programmable Logic and Applications, 2009. 
[19] Y. Sun, P. Li, G. Gu, Y. Wen, Y. Liu, , D. Liu, HMMER Acceleration Using Systolic Array Based Reconfigurable Architecture, in: IEEE International Workshop on High Performance Computational Biology, 2009.

[20] J. F. Eusse Giraldo, N. Moreano, R. P. Jacobi, A. C. M. A. de Melo, A hmmer hardware accelerator using divergences, in: Proceedings of the Conference on Design, Automation and Test in Europe, 2010, pp. 405-410. URL http://portal.acm.org/citation.cfm?id=1870926.1871023

[21] R. B. Batista, A. Boukerche, A. C. M. A. de Melo, A parallel strategy for biological sequence alignment in restricted memory space, J. Parallel Distrib. Comput. 68 (2008) 548-561. doi:10.1016/j.jpdc.2007.08.007. URL http://portal.acm.org/citation. cfm?id=1347461.1347729

[22] D. R. Horn, M. Houston, P. Hanrahan, ClawHMMER: A streaming hmmer-search implementation, SC Conference 0 (2005) 11. doi:http: //doi.ieeecomputersociety.org/10.1109/SC.2005.18.

[23] N. Ganesan, R. D. Chamberlain, J. Buhler, M. Taufer, Accelerating hmmer on gpus by implementing hybrid data and task parallelism, in: Proceedings of the First ACM International Conference on Bioinformatics and Computational Biology, BCB '10, ACM, New York, NY, USA, 2010, pp. 418-421. doi:http://doi.acm.org/10.1145/1854776.1854844.

URL http://doi.acm.org/10.1145/1854776.1854844

[24] S. R. Eddy, A new generation of homology search tools based on probabilistic inference, International Conference on Genome Informatics 23 (2009) 205-211.

[25] Gautam, S. Rajopadhye, Simplifying reductions, in: POPL '06: Conference record of the 33rd ACM SIGPLAN-SIGACT symposium on Principles of programming languages, ACM, New York, NY, USA, 2006, pp. 30-41. doi: http://doi.acm.org/10.1145/1111037.1111041. 
[26] R. E. Ladner, M. J. Fischer, Parallel Prefix Computation, Journal of ACM 27 (4) (1980) 831-838. doi:http://doi.acm.org/10.1145/322217. 322232 .

[27] S. Knowles, A family of adders, in: ARITH '99: Proceedings of the 14th IEEE Symposium on Computer Arithmetic, IEEE Computer Society, Washington, DC, USA, 1999, p. 30.

[28] R. Brent, H. Kung, A regular layout for parallel adders, IEEE Transactions on Computers C-31 (3) (1982) 260 -264. doi:10.1109/TC.1982.1675982.

[29] P. M. Kogge, H. S. Stone, A parallel algorithm for the efficient solution of a general class of recurrence equations, IEEE Transcation on Computers 22 (8) (1973) 786-793. doi:http://dx.doi.org/10.1109/TC.1973.5009159.

[30] T. Han, D. A. Carlson, Fast area-efficient vlsi adders (1987).

[31] J. Sklansky, Conditional-sum addition logic, IRE Transactions on Electronic Computers EC-9 (2) (1960) 226 -231. doi:10.1109/TEC.1960. 5219822

[32] D. Harris, A taxonomy of parallel prefix networks, in: Thirty-Seventh Asilomar Conference on Signals, Systems and Computers, Vol. 2, 2003, pp. 2213 - 2217 Vol.2. doi:10.1109/ACSSC.2003.1292373.

[33] J. Allred, J. Coyne, W. Lynch, V. Natoli, J. Grecco, J. Morrissette, SmithWaterman implementation on a FSB-FPGA module using the Intel Accelerator Abstraction Layer, in: IPDPS, IEEE, 2009, pp. 1-4.

[34] C.-I. research group, The gecos: The generic compiler suite, http://gecos.gforge.inria.fr/.

[35] M. Sheeran, Functional and dynamic programming in the design of parallel prefix networks, Journal of Functional Programming 21 (2011) 59-114. 
[36] S. Derrien, P. Quinton, Hardware acceleration of hmmer on fpgas, Journal of Signal Processing Systems 58 (2010) 53-67, 10.1007/s11265-008-0262-y. URL http://dx.doi.org/10.1007/s11265-008-0262-y

[37] T. F. Oliver, L. Y. Yeow, B. Schmidt, High performance database searching with hmmer on fpgas, in: IPDPS, IEEE, 2007, pp. 1-7. 
Table 1: Performance in GCUPS for Pfam-B.fasta

\begin{tabular}{|l|c|c|c|c|}
\hline HMMER & $\begin{array}{c}\text { globin.hmm, } \\
\mathrm{M}=143\end{array}$ & $\begin{array}{c}\text { Pkinase.hmm, } \\
\mathrm{M}=255\end{array}$ & $\begin{array}{c}\text { rrm.hmm, } \\
\mathrm{M}=77\end{array}$ & $\begin{array}{c}\text { fn3.hmm, } \\
\mathrm{M}=84\end{array}$ \\
\hline V2 & $\approx 0.03$ & $\approx 0.03$ & $\approx 0.03$ & $\approx 0.03$ \\
\hline V3-noSSE & 0.3 & 0.37 & 0.3 & 0.26 \\
\hline V3-SSE & 5.2 & 7.16 & 2.83 & 2.65 \\
\hline
\end{tabular}


Table 2: Reported average performance for previous FPGA implementation of HMMER2

\begin{tabular}{|l|c|c|}
\hline & Min GCUPS & Max GCUPS \\
\hline Takagi [18] & 0.78 & 7.38 \\
\hline Y Sun [19] & 0.28 & 3.2 \\
\hline T. Oliver [16] & & 5.3 \\
\hline
\end{tabular}


Table 3: Characteristics of various parallel-prefix networks

\begin{tabular}{|c|c|c|}
\hline Method & Delay & Cost \\
\hline Sklansky[31] & $\log _{2} N$ & $\frac{N}{2} \log _{2} N$ \\
\hline Ladner-Fischer [26] & $\log _{2} N+1$ & $\frac{N}{4}\left(\log _{2} N-1\right)+N$ \\
\hline Kogge-Stone [29] & $\log _{2} N$ & $N \log _{2} N-N+1$ \\
\hline Brent-Kung [28] & $2 \log _{2} N-1$ & $2 N-2-\log _{2} N$ \\
\hline Han-Carlson [30] & $\log _{2} N+1$ & $\frac{N}{2} \log _{2} N+\frac{N}{4}$ \\
\hline
\end{tabular}


Table 4: A summary of the different architectural solutions, along with their space-time characteristics

\begin{tabular}{|c|c|c|c|}
\hline Method & Area & $T_{c l k}$ & Through-put \\
\hline Combinational & $O(M)$ & $O\left(\log _{2} M\right)$ & $O\left(\frac{M}{\log _{2} M}\right)$ \\
\hline Tiled & $O(M / P)$ & $O\left(\log _{2} \frac{M}{P}\right)$ & $O\left(\frac{M}{\log _{2} \frac{M}{P}}\right)$ \\
\hline C-slow & $O(M)$ & $O(1)$ & $O(M)$ \\
\hline Tiled + C-slow & $O(M / P)$ & $O(1)$ & $O(M / P)$ \\
\hline
\end{tabular}


Table 5: Speed and resource usage of Single MSV kernel hardware implementation

\begin{tabular}{|c|c|c|c|c|c|}
\hline $\mathrm{M}$ & C-Slow $(S)$ & Logic Util. & M9K & MHz & GCUPS \\
\hline \hline 64 & 7 & $10 \mathrm{k} / 5 \%$ & $66 / 8 \%$ & 215 & 14 \\
\hline 128 & 8 & $19 \mathrm{k} / 9 \%$ & $130 / 15 \%$ & 201 & 25 \\
\hline 256 & 9 & $37 \mathrm{k} / 19 \%$ & $258 / 30 \%$ & 175 & 45 \\
\hline 512 & 10 & $69 \mathrm{k} / 34 \%$ & $513 / 60 \%$ & 160 & 81 \\
\hline
\end{tabular}


Table 6: Speed/Area results for combinational parallel max prefix implementations on StratixII FPGA

\begin{tabular}{|c|c|c|c|c|}
\cline { 2 - 5 } \multicolumn{1}{c|}{} & \multicolumn{4}{c|}{ Circuit Size } \\
\hline Method & 16 & 32 & 64 & 128 \\
\hline \multicolumn{5}{|c|}{ Area in LUTs } \\
\hline Brent-Kung & 694 & 1511 & 3152 & 6488 \\
\hline Sklansky & 512 & 2728 & 6266 & 14226 \\
\hline Kogge-Stone & 1846 & 4637 & 11394 & 26786 \\
\hline Ladner-Fischer & 921 & 2179 & 4963 & 11044 \\
\hline Han Carlson & 1109 & 2805 & 6765 & 15827 \\
\hline \multicolumn{5}{|c|}{$F_{\max }$ in MHz } \\
\hline Brent-Kung & 82 & 59.7 & 47.24 & 36.91 \\
\hline Ladner-Fischer & 109 & 79.6 & 65.6 & 53.4 \\
\hline Kogge-Stone & 102 & 76.8 & 61 & 46.8 \\
\hline Ladner-Fischer & 73 & 59 & 47 & 38 \\
\hline Han Carlson & 73 & 53 & 41 & 32 \\
\hline
\end{tabular}


Table 7: Performance and area for a Single P7Viterbi implementation, $S=\log _{2}(M)$

\begin{tabular}{|c|c|c|c|c|c|}
\hline $\mathrm{P}$ & $\mathrm{M}$ & Logic Util. & M9K & $\mathrm{MHz}$ & GCUPS \\
\hline \hline 8 & 64 & $5.8 \mathrm{~K} / 2.8 \%$ & $69 / 8 \%$ & 126 & 1 \\
\hline 8 & 128 & $6.8 \mathrm{~K} / 3.3 \%$ & $128 / 14.8 \%$ & 124 & 0.99 \\
\hline 16 & 64 & $10.1 \mathrm{~K} / 4.9 \%$ & $112 / 13 \%$ & 119 & 1.9 \\
\hline 16 & 256 & $14 \mathrm{~K} / 6.9 \%$ & $170 / 19.7 \%$ & 117 & 1.87 \\
\hline 32 & 256 & $28.7 \mathrm{k} / 14 \%$ & $332 / 38 \%$ & 112 & 3.6 \\
\hline
\end{tabular}


Table 8: Speed/Area results for our System-Level implementation

\begin{tabular}{|c|c|c|c|c|c|c|}
\hline $\mathrm{M}$ & $\mathrm{P}$ & Logic Util. & M9K & MLAB & MHz & GCUPS \\
\hline \hline 64 & 8 & $21 \mathrm{~K} / 10 \%$ & $54 / 6 \%$ & $27 \mathrm{~Kb}$ & 99 & 7 \\
\hline 64 & 16 & $26 \mathrm{~K} / 13 \%$ & $99 / 11 \%$ & $29 \mathrm{~Kb}$ & 97 & 7.6 \\
\hline 128 & 8 & $31 \mathrm{~K} / 15 \%$ & $57 / 7 \%$ & $51 \mathrm{~Kb}$ & 94 & 12.7 \\
\hline 128 & 16 & $38 \mathrm{k} / 19 \%$ & $105 / 12 \%$ & $55 \mathrm{~Kb}$ & 93 & 13 \\
\hline 512 & 8 & $79 \mathrm{~K} / 39 \%$ & $675 / 78 \%$ & $66 \mathrm{~Kb}$ & 89 & 45.7 \\
\hline
\end{tabular}


Table 9: Performance and area for our System-Level implementation

\begin{tabular}{|c|c|l|l|c|c|c|c|c|}
\hline M & P & MSV & P7Vit & Logic Util. & M9K & MLAB & MHz & GCUPS \\
\hline \hline 64 & 8 & 6 & 1 & $128 \mathrm{~K} / 63 \%$ & $864 / 100 \%$ & $215 \mathrm{~Kb}$ & 103 & 40.0 \\
\hline 64 & 8 & 7 & 1 & $143 \mathrm{~K} / 70 \%$ & $864 / 100 \%$ & $269 \mathrm{~Kb}$ & 97 & 44.2 \\
\hline 128 & 8 & 3 & 1 & $105 \mathrm{~K} / 52 \%$ & $864 / 100 \%$ & $181 \mathrm{~Kb}$ & 95 & 37.2 \\
\hline 256 & 8 & 2 & 1 & $147 \mathrm{~K} / 72 \%$ & $864 / 100 \%$ & $203 \mathrm{~Kb}$ & 99 & 51.5 \\
\hline 512 & 8 & 1 & 1 & $79 \mathrm{~K} / 39 \%$ & $675 / 78 \%$ & $66 \mathrm{~Kb}$ & 89 & 45.7 \\
\hline
\end{tabular}




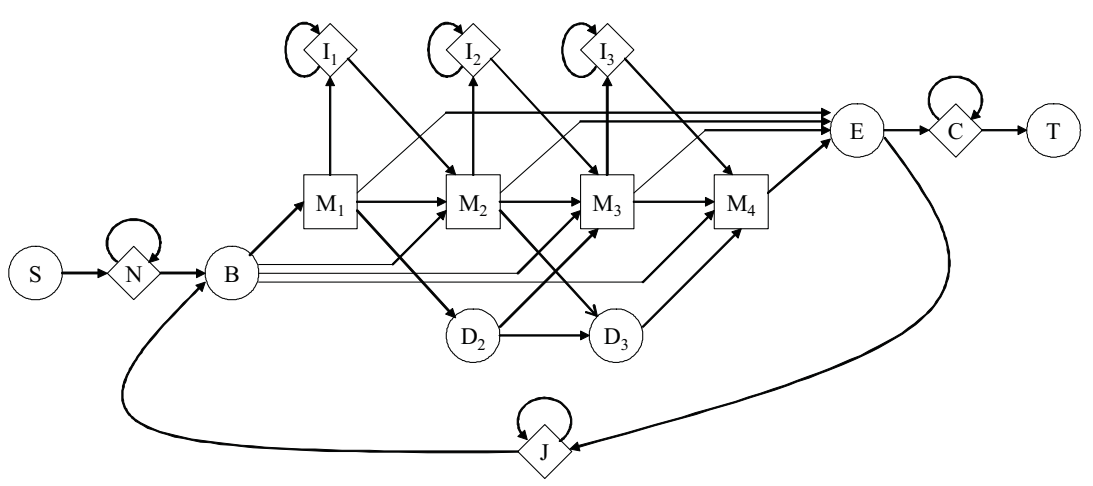

Figure 1: Structure of a Plan7 HMM 37. 


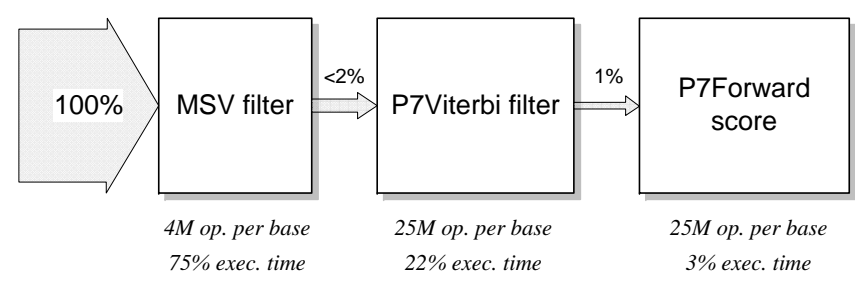

Figure 2: HMMER3 execution pipeline, with profiling data 


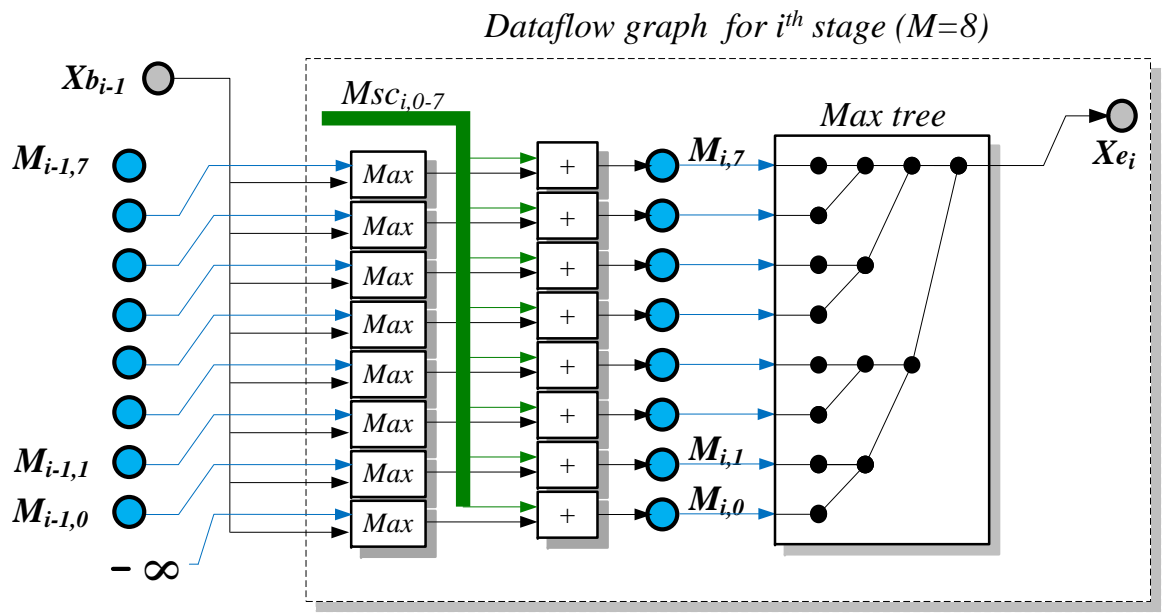

Figure 3: Dataflow dependencies for one stage of the MSV filter $(M=8)$ algorithm after rewriting 


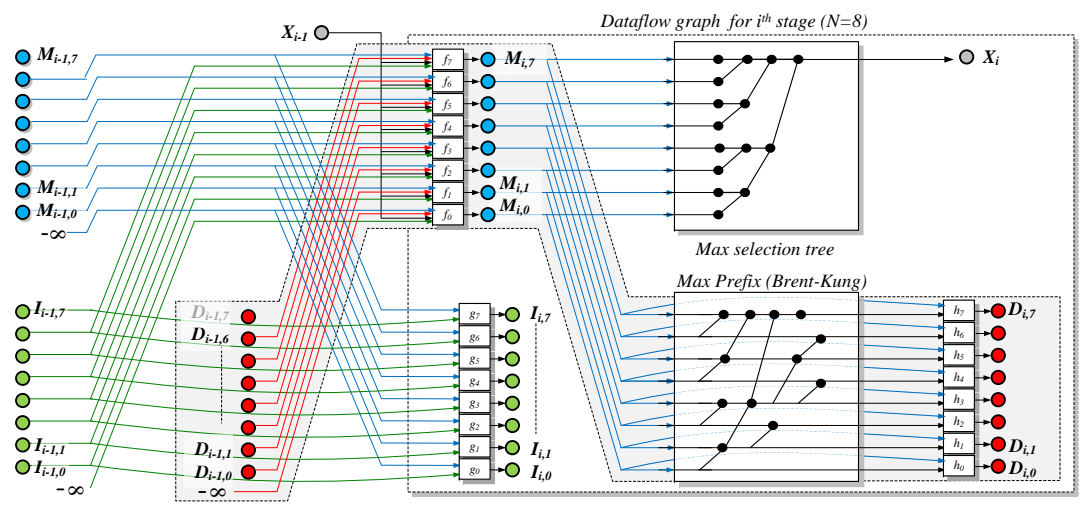

Figure 4: Dataflow dependencies for one stage ${ }_{\boldsymbol{I}_{i, 7}}$ the P7Viterbi $\left(N=8 p_{i, \text { algorithm }}\right.$ after rewriting. The dependency $D_{i, k} \longrightarrow D_{i, k-1}$ in equation 3 is converted to a max-prefix block, reducing the critical path from $O(N)$ to $O\left(\log _{2} N\right)$ operations. 


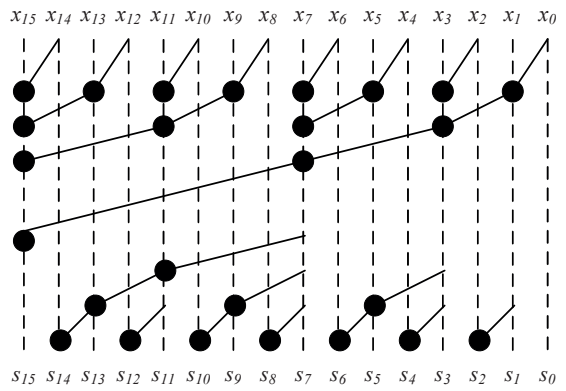

(a) Brent-Kung

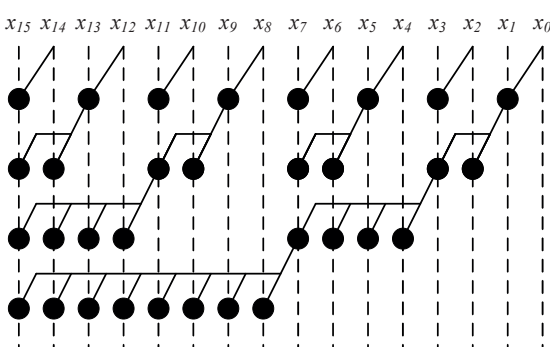

(b) Sklansky

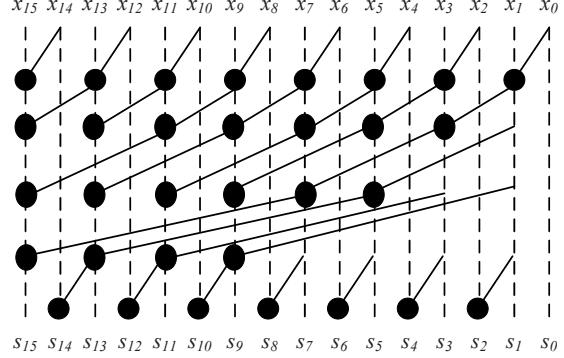

(c) Han Carlson

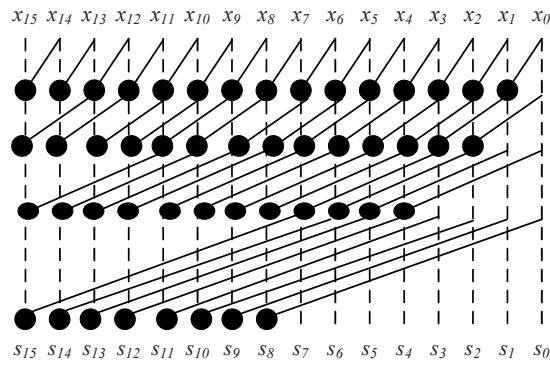

(d) Kogge-Stone

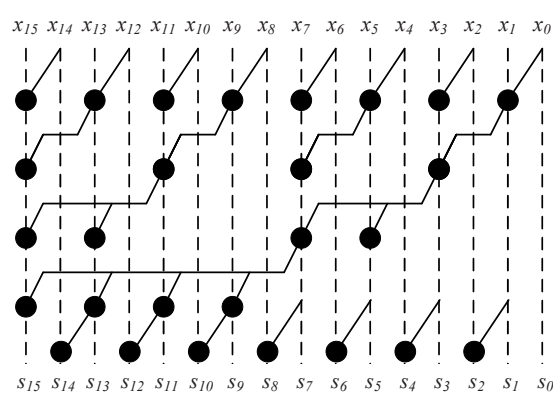

(e) Ladner-Fischer

Figure 5: Examples of parallel prefix implementation for $N=16$ 


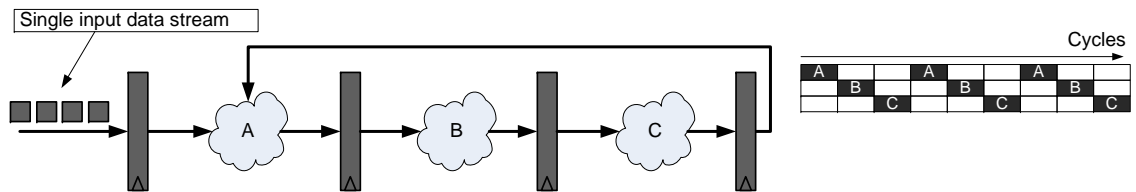

(a) Pipelining without C-slow: The pipeline with a single input data stream and a feedback loop from end to the start will not be completely filled at any time instance due to dependency on previous results and at each cycle only one logic block will be active.

Multiple independent input data stream

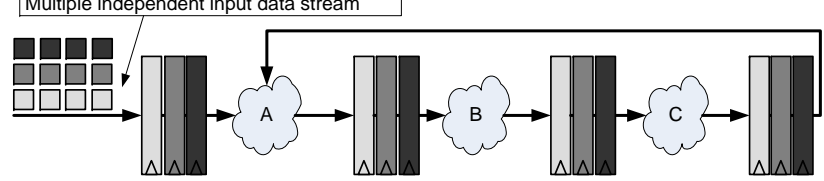

Cycles

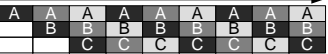

(b) Pipelining with C-slow: In presence of a feedback loop in a pipeline, several (precisely equal to number of pipeline stages inside feedback loop) independent interleaved input data streams make efficient use of pipeline hardware. Now all logic block are active at each cycle and operating on independent data streams.

Figure 6: Impact of pipelining with C-slow in presence of a feedback loop 

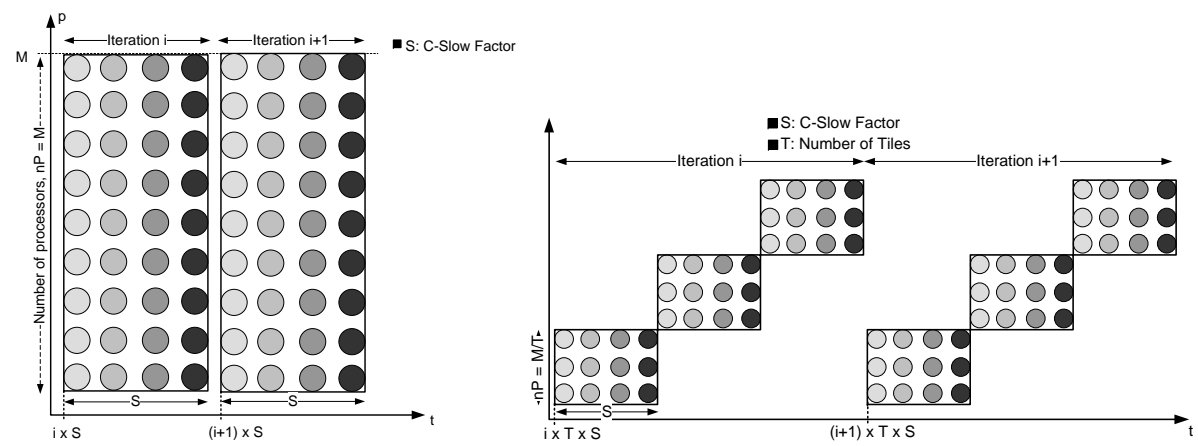

(a) Viterbi with C-slow: A Viterbi ker- (b) Viterbi with C-slow and tiling: A viterbi kernel nel with $S$ pipeline stages is receiving $S$ implemented with $S$ pipeline stages and $S$ interleaved independent interleave data streams, to independent data inputs on $M / T$ processors through overcome feedback loop effect. The re- tiling.

quired number of processors is $\mathrm{M}$ (i.e.,

the profile size).

Figure 7: Viterbi kernel implementation with simple C-Slowed pipeline and Tiled C-slowed pipeline 


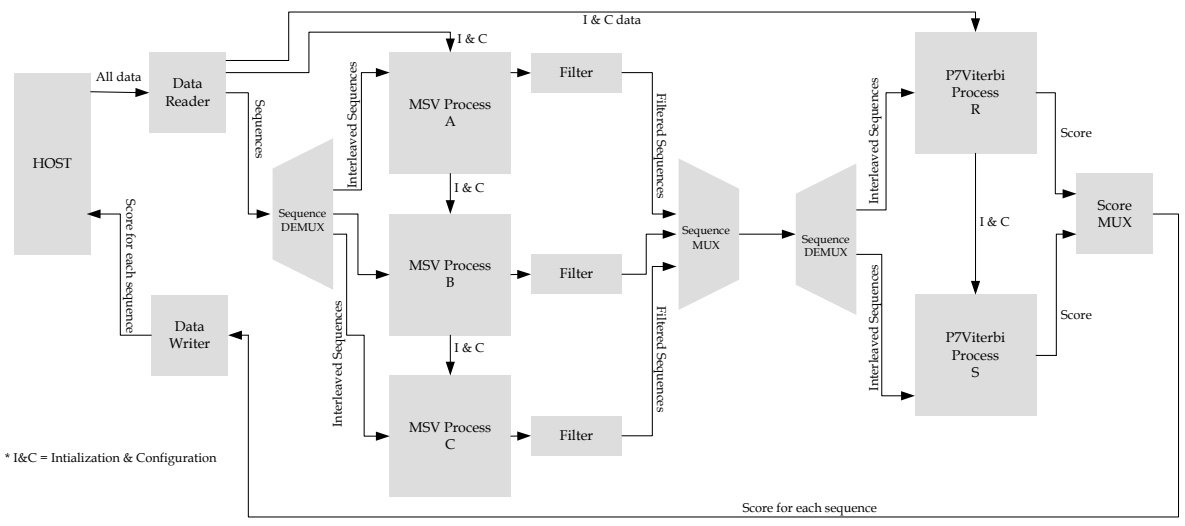

Figure 8: System level view of a complete HMMER3 hardware accelerator: The figure shows an HMMER3 pipeline with 5 computation blocks (i.e., 3 MSV blocks communicating to 2 P7Viterbi blocks). The Sequence DEMUX distributes the interleaved sequences between computation blocks. The Filter filters out the sequences with scores lower than the threshold. The Sequnce MUX receives interleaved sequences from different sources, it interleaves all incoming sequences and send to Sequence DEUMX. The Sequence DEUMX will redistribute the input sequences according to the number of PEs and CSlow factor S within each PE. 


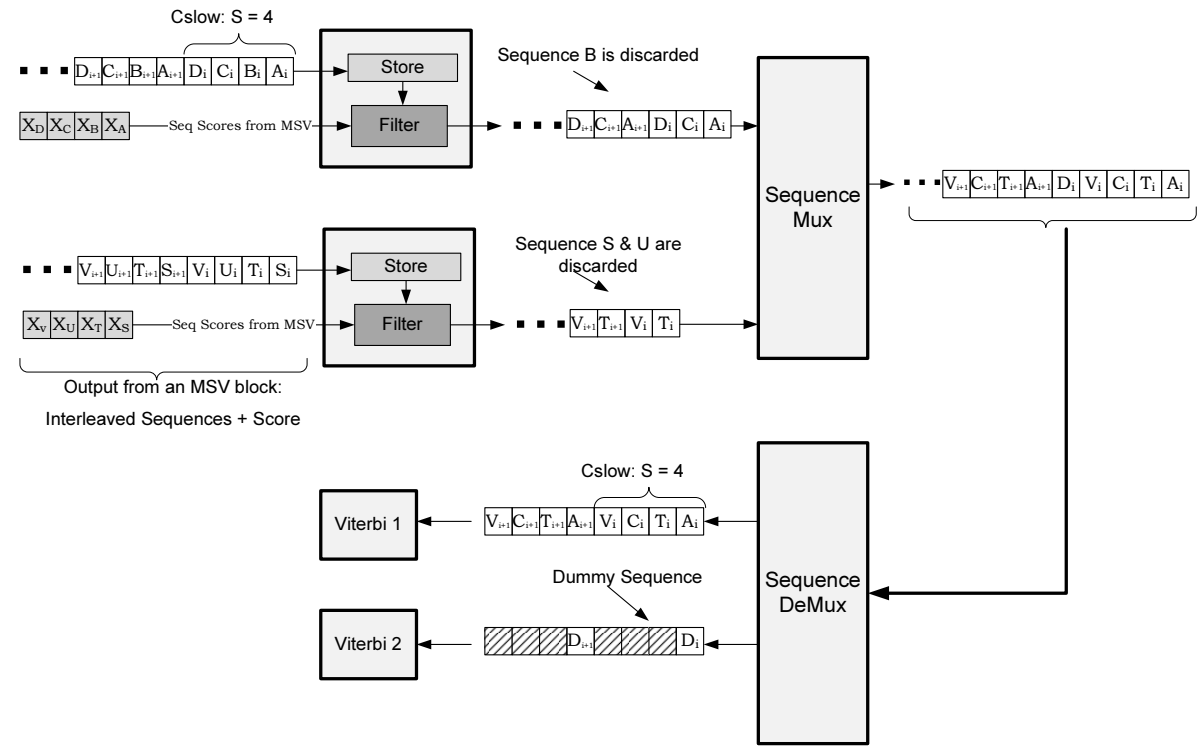

Figure 9: Sequence Handling within Pipeline: The figure shows handling \& filteration of sequences after MSV computation with 2 MSV blocks communicating to 2 P7Viterbi blocks). The CSlow factor 'S' $=4$. 'A','B','C', 'D' and 'S', 'T', 'U',' $V$ ' are respective interleaved sequences coming from MSV block alongwith respective scores, $X_{A} \ldots X_{V}$. The subscript 'i' and ' $\mathrm{i}+1$ ' reprensents the sequence characters. 

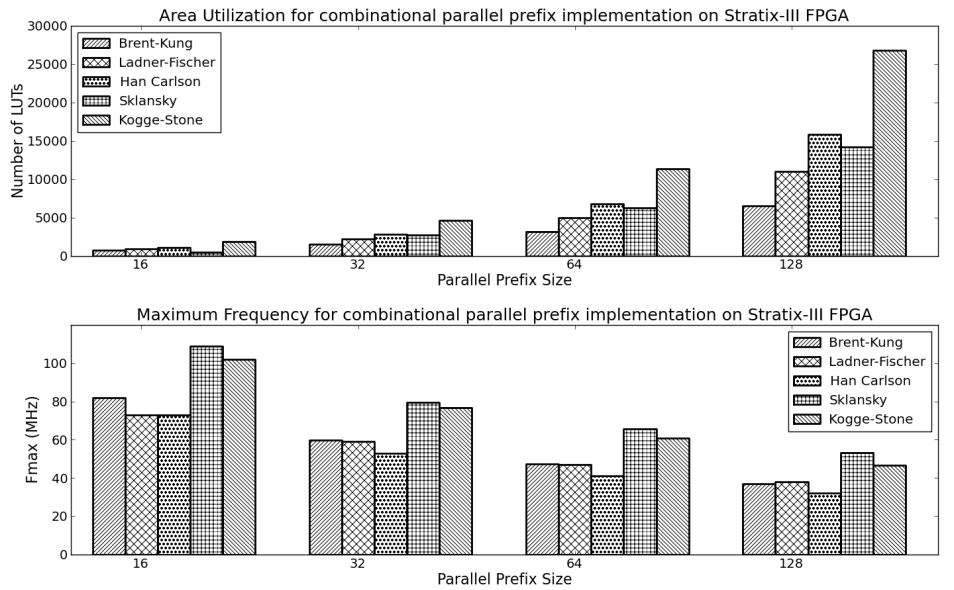

Figure 10: Speed/Area results for combinational parallel max prefix implementations on Stratix-II FPGA 

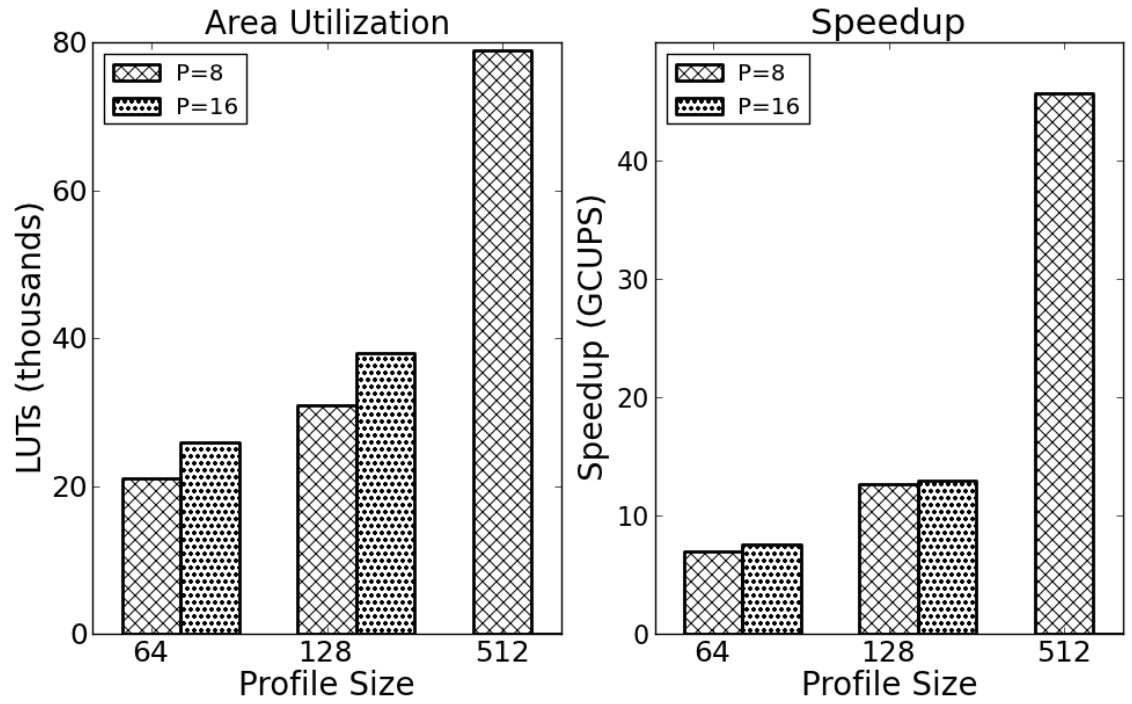

Figure 11: Speed/Area results for single HMMER3 pipeline implementation 


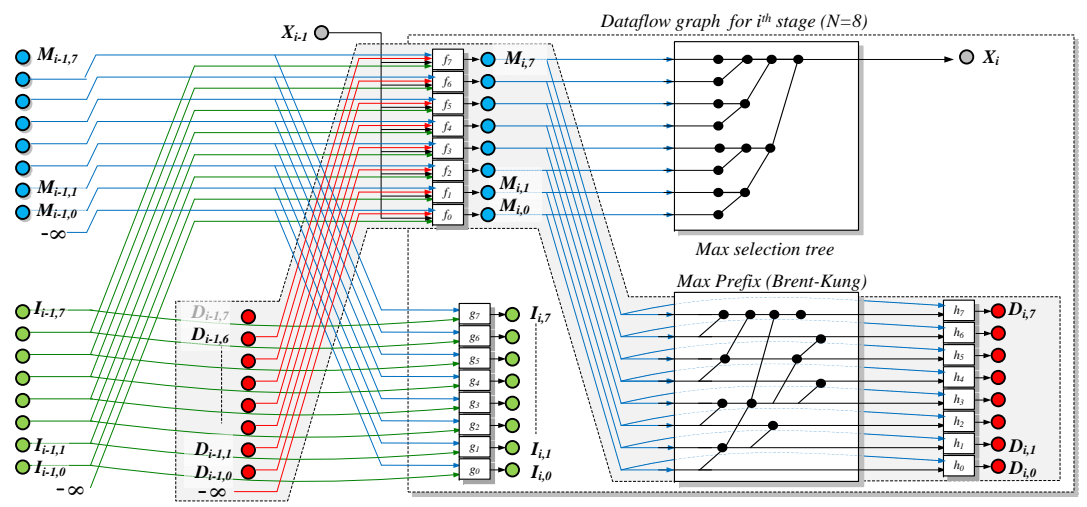

Figure 4: Dataflow dependencies for one stage ${ }_{\boldsymbol{I}_{i, 7}}$ the P7Viterbi $\left(N=8 p_{i, \text { algorithm }}\right.$ after rewriting. The dependency $D_{i, k} \longrightarrow D_{i, k-1}$ in equation (3) is converted to a max-prefix block, reducing the critical path from $O(N)$ to $O\left(\log _{2} N\right)$ operations. 


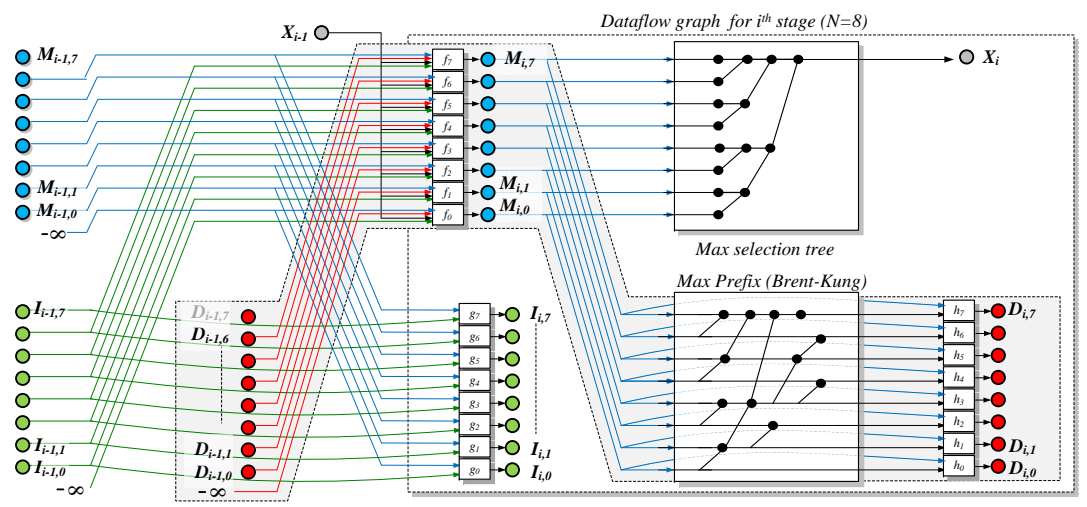

Figure 4: Dataflow dependencies for one stage ${ }_{\boldsymbol{I}_{i, 7}}$ the P7Viterbi $\left(N=8 p_{i, \text { algorithm }}\right.$ after rewriting. The dependency $D_{i, k} \longrightarrow D_{i, k-1}$ in equation (3) is converted to a max-prefix block, reducing the critical path from $O(N)$ to $O\left(\log _{2} N\right)$ operations. 


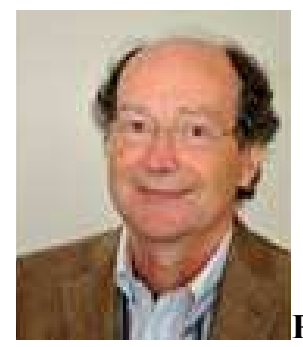

Patrice Quinton obtained a degree of Engineer in Computer Science of ENSIMAG (Grenoble, France), in 1972, and a These d'Etat in Mathematics of the University of Rennes (France) in 1980. He has been Directeur de Recherches of the CNRS, and head of the VLSI Parallel Architectures group of IRISA in Rennes between 1982 and 1997, and since then, he is professor of the University of Rennes 1. Patrice Quinton is currently deputy director of the brittany branch of Ecole Normale Suprieure of Cachan and member of the Cairn research group at IRISA, Rennes. His interests include parallel architectures, VLSI, systolic arrays, computer aided design and sensor networks. Patrice Quinton is co-author of one book, and author and co-author of about one hundred journal papers, international conference communications or book chapters.

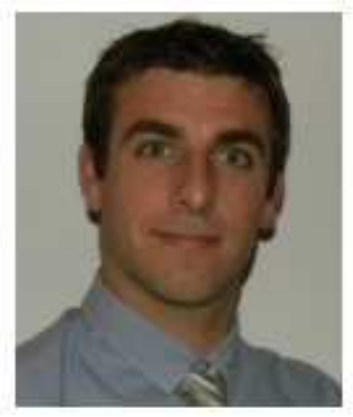

Alexandre Cornu has worked as electronics and computer science expert during three years in IRISA. He has worked on several FPGA-based architectures and on software parallelization to speed-up compute-intensive software.

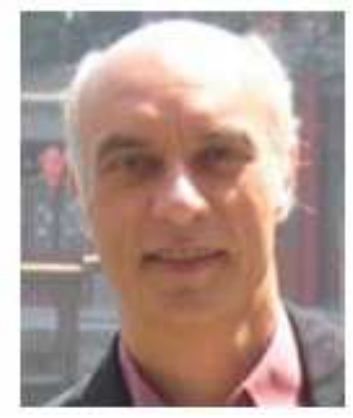

Dominique Lavenier is a senior CNRS (French National Center for Scientific Research) researcher and Professor at ENS Cachan, Brittany. He is currently leading the Symbiose bioinformatics team at IRISA/INRIA, Rennes His research interests include HPC, Parallel architecture, GPU Computing Bioinformatics, Structural Biology and the processing of genomic data coming from Next Generation Sequencing technologies. 